\title{
Multiple Myeloma Mimics Bone Metastasis From a Rectal Adenocarcinoma
}

\author{
Im-Kyung Kim, Jeonghyun Kang, Yu Ri Kim, Tae Joo Jeon², Seung Hyuk Baik, Seung-Kook Sohn \\ Departments of Surgery, ${ }^{1}$ Internal Medicine, and ${ }^{2}$ Nuclear Medicine, Gangnam Severance Hospital, Yonsei University College of Medicine, \\ Seoul, Korea
}

A presumptive diagnosis of bone metastasis can be easily made when a patient with a history of colorectal cancer develops bone lesions that are seen on follow-up imaging. In this case report, we describe a patient whose multiple bone lesions were wrongly attributed to a recurrence of rectal cancer rather than being identified as multiple myeloma lesions. When clinicians detect new, abnormal, bony lesions in a patient with a previous history of cancer, they should consider diseases such as multiple myeloma in their differential diagnosis.

Keywords: Multiple myeloma; Bone metastasis; Rectal neoplasms

\section{INTRODUCTION}

Skeletal bone is a frequent site of distant metastasis in patients with advanced solid cancers, such as breast, prostate, and lung cancers [1]. In contrast, primary colorectal cancer rarely metastasizes to the bone late in its course [2,3]. Regardless, a presumptive diagnosis of bone metastasis can be easily made when a patient with a history of colorectal cancer develops bone lesions that are seen on follow-up imaging.

Multiple myeloma (MM) is a malignant neoplasm of plasma cells and mainly involves the bone marrow; it accounts for approximately $10 \%$ of hematologic malignancies [4]. The overall survival of patients with MM has improved significantly in the last decade with the emergence of thalidomide, bortezomib, lenalidomide, and hematopoietic stem-cell transplantation [4]. Although plain radiographs of the skeleton are routinely used to assess the extent of bone involvement, positron emission tomography/computed tomography (PET/CT) and magnetic resonance imaging (MRI) are frequently added to increase the sensitivity or

Received: October 27, 2016 - Accepted: January 2, 2017

Correspondence to: Jeonghyun Kang, M.D.

Department of Surgery, Gangnam Severance Hospital, Yonsei University College of Medicine, 211 Eonju-ro, Gangnam-gu, Seoul 06273, Korea Tel: +82-2-2019-3369, Fax: +82-2-3462-5994, E-mail: ravic@naver.com

(C) 2017 The Korean Society of Coloproctology

This is an open-access article distributed under the terms of the Creative Commons Attribution NonCommercial License (http://creativecommons.org/licenses/by-nc/4.0) which permits unrestricted noncommercial use, distribution, and reproduction in any medium, provided the original work is properly cited. specificity of the diagnosis $[4,5]$.

The coexistence of MM and colon cancer has rarely been reported in the literature [6-9]. Because of their distinct treatment modalities, the ability of clinicians to distinguish MM from bone metastasis secondary to colorectal cancer is important. However, previous reports have demonstrated that despite advanced imaging studies, discrimination between MM and bone metastasis from colon cancer is difficult $[8,9]$. For that reason, this case report describes a patient whose multiple bone lesions were wrongly attributed to a recurrence of rectal cancer rather than being identified as MM lesions. This misunderstanding resulted in a delay of appropriate treatment.

\section{CASE REPORT}

A 69-year-old male patient visited an outpatient clinic for his fifth annual follow-up. He had a history of rectal cancer for which he had undergone a long course of preoperative chemoradiotherapy, followed by a low anterior resection with colo-anal anastomosis. The pathology of the surgical specimen revealed proper muscle invasion without any regional or distant metastases (ypT2N0M0; yp stage I). Adjuvant chemotherapy, based on a regimen containing 5-fluorouracil (FU), was given 4 times.

At the fifth annual follow-up, the following tests were performed: serum carcinoembryonic antigen (CEA), colonoscopy, pelvic MRI, and PET/CT. The colonoscopy showed no evidence of local recurrence. The patient's CEA level was $1.5 \mathrm{ng} / \mathrm{mL}$. However, pelvic MRI revealed multiple, newly developed, small nod- 
Volume 33, Number 2, 2017

ules in the pelvic bones that were suggestive of bony metastases (Fig. 1). The PET/CT scan also showed multiple hypermetabolic foci in the bilateral humerus, femurs, scapulae, ribs, spine and pelvic bones (Fig. 2). Further study using Tc-99m methylene diphosphonate (MDP) whole-body bone images showed increased uptake in the right anterior sixth rib, the spinous process of T9, and the upper portion of the bilateral sacroiliac joints (Fig. 3).

Under a presumptive diagnosis of bone metastases from rectal cancer, palliative chemotherapy with the XELOX regimen

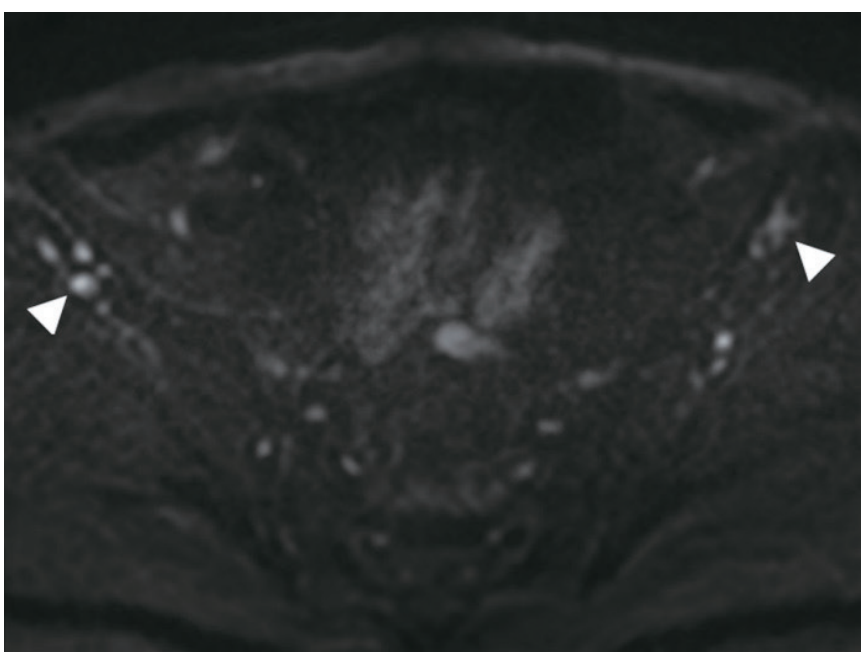

Fig. 1. Pelvic magnetic resonance imaging revealed newly developed, multiple, small, nodular lesions in the pelvic bone (white arrowheads).

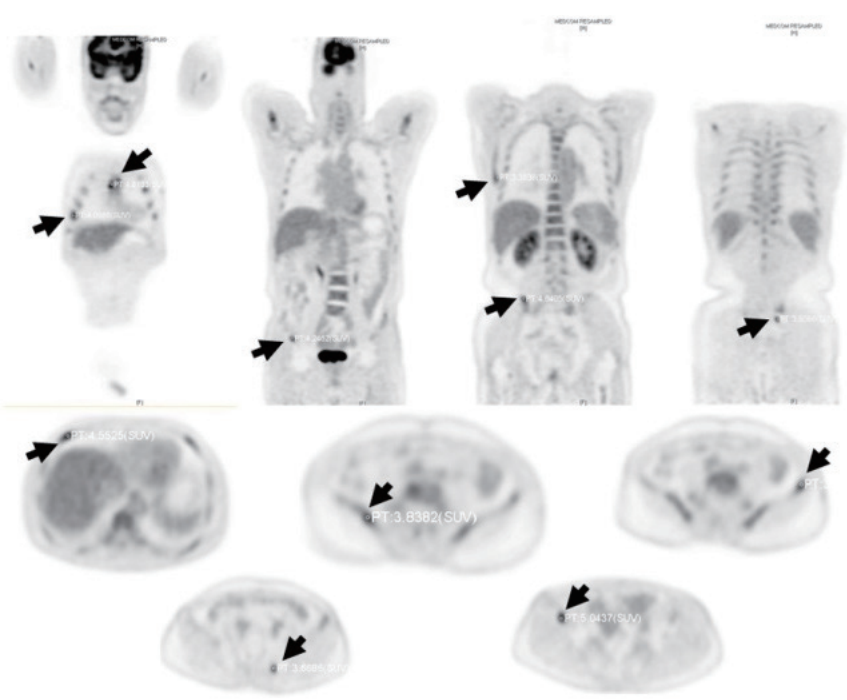

Fig. 2. Positron emission tomography/computed tomography scans demonstrated newly developed, multiple, hypermetabolic foci in the bilateral humerus, femurs, scapulae, ribs, spine and pelvic bones (black arrows). (capecitabine, 5-FU and oxaliplatin) was planned. However, chemotherapy was delayed for approximately five weeks due to abnormal laboratory findings, including anemia, leukocytopenia and mildly-elevated serum creatinine $(\mathrm{Cr})$. After the first dose of XELOX chemotherapy, the patient was noted to have pancytopenia and a gradually-rising serum $\mathrm{Cr}$ level. The patient also suffered from poor oral intake and nausea. These clinical manifestations and abnormal laboratory findings were attributed to the adverse effects of XELOX chemotherapy. Therefore, further chemotherapy could not be given. The pancytopenia and the elevated serum $\mathrm{Cr}$ persisted for 2 months after the last chemotherapy dose. The patient was transferred to the Department of Nephrology to evaluate his elevated Cr levels. Initially, the patient was suspected to have contrast-induced acute kidney injury or chemotherapyinduced chronic renal disease. A 24-hour urine study detected a large amount of protein. Urinary immunoelectrophoresis demonstrated a monoclonal gammopathy with an M spike. Furthermore, urinary immunofixation electrophoresis revealed an abnormal band in the kappa lane. Serum protein electrophoresis showed hypogammaglobulinemia $(680 \mathrm{mg} / \mathrm{dL})$ with an elevated free kappa light chain $(6,090 \mathrm{mg} / \mathrm{L})$.

The above results led us to perform a bone marrow biopsy for suspected MM. The biopsy revealed hypercellular marrow particles with marked plasmacytosis $(88.3 \%$ of absolute neutrophil counts). Based on these results, a final diagnosis of MM (kappa

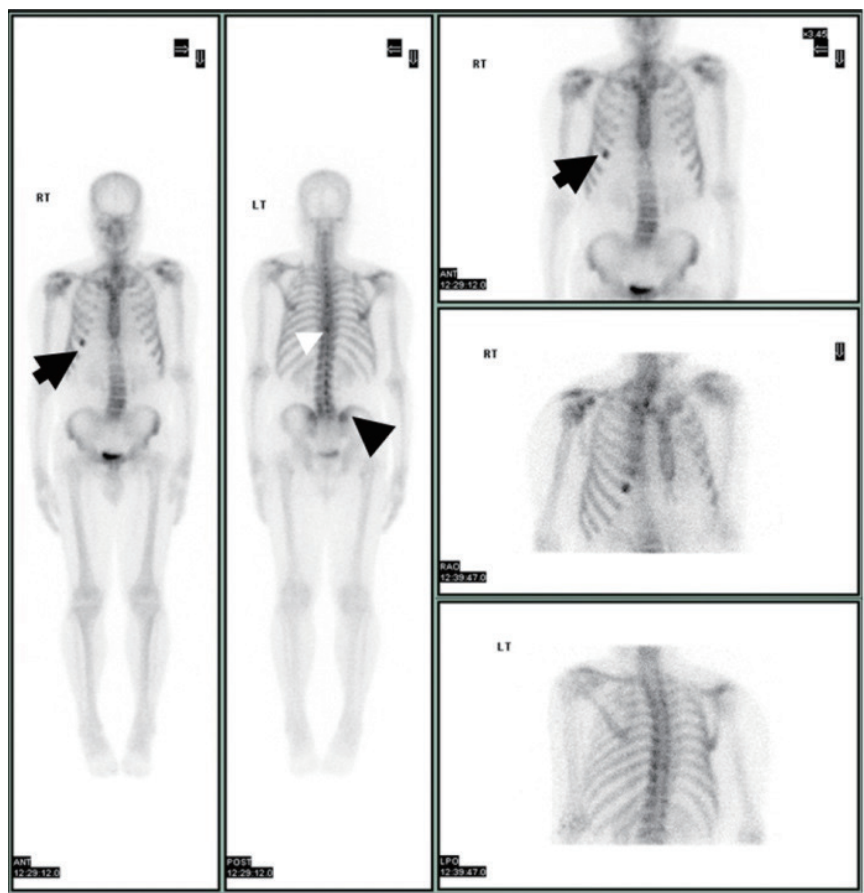

Fig. 3. Tc-99m methylene diphosphonate showed increased uptakes in the right anterior sixth rib (black arrow), spinous process of T9 (white arrowhead), and upper portion of the bilateral Sacroiliac joints (black arrowhead). 
type) was made. Chemotherapy was initiated with a combined regimen of bortezomib, melphalan and prednisone (VMP regimen) every 35 days. A 50\% reduction of in the recommended dose of mephalan and prednisone were initially administered due to chronic renal failure. During the first cycle of the VMP regimen, we reduced the dose of bortezomib by an additional $75 \%$ because the patient experienced severe diarrhea. After the fifth cycle of chemotherapy, the hypogammaglobulinemia $(720 \mathrm{mg} /$ $\mathrm{dL}$ ) with free kappa light chain elevation (587 mg/L) improved, suggestive of a partial response. Eight cycles of the VMP regimen were completed. The patient was offered an additional 12 cycles of cyclophosphamide, thalidomide and dexamethasone (CTD regimen). However, the disease had progressed. Unfortunately, the patient could not tolerate further chemotherapy (including low dose dexamethasone with lenalidomide) and was lost to follow up.

\section{DISCUSSION}

A few prior studies have reported the coexistence of $\mathrm{MM}$ and colon cancer [6-9]. However, to the best of our knowledge, this is the first case of MM mimicking bone metastases from rectal cancer. In this case, a presumptive diagnosis was made based on the patient's history of rectal cancer and the presence of multiple bony lesions on imaging.

MM causes approximately $1 \%$ of all malignancies and is increasing in prevalence annually. Most patients undergo an asymptomatic period, defined as monoclonal gammopathy of undetermined significance (MGUS). At a rate of approximately $1 \%$ per year, MGUS progresses to myeloma [4]. MM may present with the following signs of end-organ damage: hypercalcemia, renal insufficiency, anemia, and bone lesions (including lytic lesions and osteopenia) [5]. Although the patient described here had thrombocytopenia and elevated serum $\mathrm{Cr}$, these abnormal laboratory findings are not specific to MM. Furthermore, the prolonged pancytopenia and elevated Cr were thought to have resulted from the first cycle of XELOX chemotherapy. These clinical situations resulted in a delay in the proper diagnosis and management.

Recent reports have demonstrated that most patients with bone metastasis from colorectal cancer have initially advanced stages (stage III or IV), high levels of CEA at the time of diagnosis, and a median time interval to bone metastasis of 10-11 months [2, 3]. In addition, isolated bony metastases without the involvement of another organ, such as the liver or the lungs, are rare $[2,10]$. In this case, the patient had stage I rectal cancer postoperatively, with no elevation of the CEA level (2.1 when rectal cancer was diagnosed, and 1.5 when MM was diagnosed). No other metastatic lesions, except those in the bone, were present. Furthermore, almost 5 years had elapsed between the initial resection of the primary tumor and the detection of possible recurrence. If the bony lesions were truly metastases from rectal cancer, the patient's presentation would be considered extremely rare and inconsistent with each subcategory of bone metastasis from colorectal cancer. Even though, the clinician, depending on a thorough review of radiologic imaging and multidisciplinary discussion, considered bone metastasis as a priority of diagnosis.

In the diagnosis of MM, whole body X-ray (WBXR), CT, MRI, radionuclide imaging, and PET/CT are currently used. That Tc99m MDP may not detect all lesions in MM is well known [11]. PET/CT or PET is useful in the detection of occult lesions that are invisible in Tc-99m MDP. When the Durie-Salmon PLUS staging system is used, PET/CT or PET imaging is required [5]. Although PET scans have a superior detection rate for bone lesions compared to WBXR, the superiority of PET/CT in comparison to MRI has not yet been established [5]. Shortt et al. [12] reported that when PET and whole-body MRI were used in combination with concordant results, the positive predictive value was $100 \%$.

However, data addressing the sensitivity or the specificity of diagnostic modalities in discriminating MM from bone metastasis are limited, potentially given its rarity. The incidence of bone metastases from primary colorectal cancer is relatively low (less than $10 \%$ ) compared to that of other solid tumors. The most common sites for skeletal metastases are the vertebral column, particularly the lumbar and the sacral portions, followed by the pelvic bones and ribs $[2,3,13,14]$. The radiologic findings usually demonstrate osteolytic or mixed osteolytic-osteoblastic patterns, resembling the patterns described in our case. For this reason, previous case reports have also demonstrated that distinguishing between $\mathrm{MM}$ and bone metastasis, even with the addition of PET/CT scans, is difficult $[8,9]$. Considering the diagnostic challenges, this case emphasized the importance of considering MM as a potential diagnosis in the assessment of rectal cancer patients with bone lesions. This is particularly true in patients with somewhat unusual clinical presentations.

In conclusion, this case highlights the rarity of detecting MM on routine annual follow-ups for rectal cancer, in contrast to the relatively frequent diagnosis of bone metastasis from rectal cancer. When clinicians detect new abnormal bony lesions in a patient with a previous history of cancer, they should consider diseases such as MM in their differential diagnosis.

\section{CONFLICT OF INTEREST}

No potential conflict of interest relevant to this article was reported.

\section{REFERENCES}

1. Coleman RE, Rubens RD. The clinical course of bone metastases from breast cancer. Br J Cancer 1987;55:61-6.

2. Baek SJ, Hur H, Min BS, Baik SH, Lee KY, Kim NK. The characteristics of bone metastasis in patients with colorectal cancer: a long-term report from a single institution. World J Surg 2016;40: 982-6. 
3. Santini D, Tampellini M, Vincenzi B, Ibrahim T, Ortega C, Virzi V, et al. Natural history of bone metastasis in colorectal cancer: final results of a large Italian bone metastases study. Ann Oncol 2012; 23:2072-7.

4. Rajkumar SV. Multiple myeloma: 2013 update on diagnosis, riskstratification, and management. Am J Hematol 2013;88:226-35.

5. Mihailovic J, Goldsmith SJ. Multiple myeloma: 18F-FDG-PET/ CT and diagnostic imaging. Semin Nucl Med 2015;45:16-31.

6. Shanbrom E. Multiple myeloma and coexistent carcinoma of the sigmoid colon. Am J Clin Pathol 1963;40:67-71.

7. Yermiahu T, Peiser J, Benharroch D, Ovnat A. Aggressive behavior of carcinoma of the colon associated with nonsecreting plasma cell myeloma: a case report. J Clin Gastroenterol 1989;11:5657.

8. Chang CY, Peng YJ, Shen DH, Huang WS, Cherng SC. Detection of multiple myeloma by PET/CT in a patient with colon cancer. Clin Nucl Med 2008;33:367-70.

9. Zhang H, Lv J, Lv C, Zhang H. Presentation of multiple myeloma mimicking bone metastasis from colon adenocarcinoma: a case report and literature review. Mol Clin Oncol 2016;4:31-4.

10. Roth ES, Fetzer DT, Barron BJ, Joseph UA, Gayed IW, Wan DQ. Does colon cancer ever metastasize to bone first? a temporal analysis of colorectal cancer progression. BMC Cancer 2009;9:274.

11. Wang K, Allen L, Fung E, Chan CC, Chan JC, Griffith JF. Bone scintigraphy in common tumors with osteolytic components. Clin Nucl Med 2005;30:655-71.

12. Shortt CP, Gleeson TG, Breen KA, McHugh J, O'Connell MJ, O'Gorman PJ, et al. Whole-Body MRI versus PET in assessment of multiple myeloma disease activity. AJR Am J Roentgenol 2009; 192:980-6.

13. Onesti JK, Mascarenhas CR, Chung MH, Davis AT. Isolated metastasis of colon cancer to the scapula: is surgical resection warranted? World J Surg Oncol 2011;9:137.

14. Kanthan R, Loewy J, Kanthan SC. Skeletal metastases in colorectal carcinomas: a Saskatchewan profile. Dis Colon Rectum 1999;42: 1592-7. 